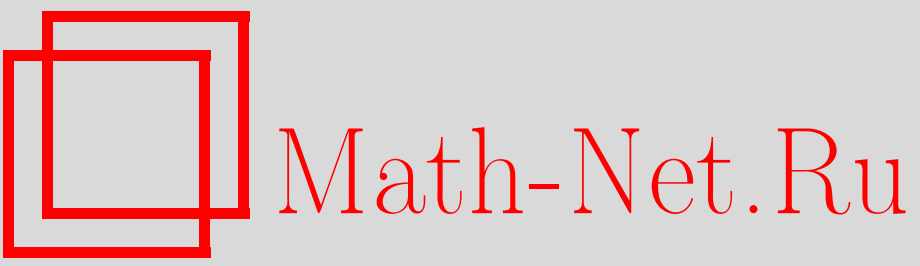

И. И. Богданов, Алгебраические расширения полуполей, УМН, 2004, том 59, выпуск 1, 181-182

DOI: https://doi.org/10.4213/rm709

Использование Общероссийского математического портала Math-Net.Ru подразумевает, что вы прочитали и согласны с пользовательским соглашением

http://www.mathnet.ru/rus/agreement

Параметры загрузки:

IP : 18.234 .197 .8

26 апреля 2023 г., 04:44:07 


\title{
АЛГЕБРАИЧЕСКИЕ РАСШИРЕНИЯ ПОЛУПОЛЕЙ
}

\author{
И. И. БОГДАНОВ
}

В заметке исследуется возможность расширения полуполя корнем алгебраического уравнения; результаты уточняются для идемпотентных и сократимых полуполей.

1. Мы используем терминологию и обозначения из [1]-[4]. Полукольцом назьвается коммутативный аддитивный моноид $(D,+, 0)$, снабженный ассоциативным дистрибутивным умножением с единицей 1 . В дальнейшем все полукольца предполагаются коммутативными. Полумо$\partial y л ь$ над полукольцом $S$ есть аддитивная полугруппа $M$, снабженная полукольцевьпм морфизмом $S \rightarrow$ End $M$, задающим действие $S$ на $M$. Полумодуль $M$ точен, если для любых различных $a, b \in S$ существует такое $m \in M$, что $a m \neq b m$. Полукольцо $S$ назьвается полуалгеброй над полукольцом $T$, если определен морфизм из $T$ в центр полукольца $S$, задающий действие $T$ на $S$.

Полукольцо называется полуполем, если любой ненулевой элемент обратим по умножению и необратим по сложению. Полуполе идемпотентно (сократимо), если сложение идемпотентно (сократимо). "Наименьшим" полуполем является $\mathbb{B}=(\{0,1\}, \max , \min )$. На любом полуполе вводится частичный порядок $x \leqslant+y \Longleftrightarrow \exists a: x+a=y$. На идемпотентном полуполе этот порядок задает решетку; при этом $x \vee y=x+y, x \wedge y=\left(x^{-1}+y^{-1}\right)^{-1}$.

Для ненулевого многочлена $P(x)=p_{n} x^{n}+\cdots+p_{0}$ введем его нижнюю степень $\operatorname{ldeg} P=$ $\min \left\{i: p_{i} \neq 0\right\}$. Уравнение $P(x)=Q(x)$ назьвается строгим, если $\operatorname{ldeg} P>\operatorname{deg} Q$ и $\operatorname{ldeg} Q=0$ (в частности, $P \neq 0 \neq Q$ ). Строгое уравнение называется мономиальным, если $\operatorname{deg} Q=0$. В работе [2] для строгого уравнения $P(x)=Q(x)$ построен его дифференциал $d[P-Q](x, y)$, однозначно определяемый следующими свойствами: (1) он билинеен, т.е. $d\left[\left(P_{1}+P_{2}\right)-Q\right]=$ $d\left[P_{1}-Q\right]+d\left[P_{2}-Q\right]$ и $d\left[P-\left(Q_{1}+Q_{2}\right)\right]=d\left[P-Q_{1}\right]+d\left[P-Q_{2}\right] ;(2) d\left[p_{n} x^{n}-q_{m} x^{m}\right](x, y)=$ $p_{n} q_{m} \sum_{i=1}^{n-m} x^{n-i} y^{m-1+i}$ при $n>m$. Показано, что существует такой многочлен $R(x, y)$, что $P(x) Q(y)+R(x, y)=x \cdot d[P-Q](x, y)$ и $R(x, y)+Q(x) P(y)=d[P-Q](x, y) \cdot y$. Отсюда следует, что у строгого уравнения не больше одного решения. Используя свойства дифференциала, можно также показать, что при любых $x, y \in D$ утверждения $P(x) Q(x)^{-1} \geqslant_{+} P(y) Q(y)^{-1}$ и $x \geqslant_{+} y$ равносильны, если уравнение $P(x)=Q(x)$ строгое.

Полуполе назовем строго (мономиально) алгебраически замкнутым, если любое строгое (мономиальное) уравнение имеет корень. Пусть $D \subseteq \widetilde{D}$ - полуполя. Если $\widetilde{D}$ строго (мономиально) алгебраически замкнуто и наименьшее строго (мономиально) алгебраически замкнутое подполуполе, содержащее $D$, совпадает с $\widetilde{D}$, то $\widetilde{D}$ назовем строгим (мономиальныц ) алгебраическим замыканием полуполя $D$.

2. Естественно возникает вопрос о возможности расширения полуполя корнем уравнения $P(x)=Q(x)$. Это расширение можно строить одним из двух эквивалентных способов:

$$
\varphi_{P=Q}: D \hookrightarrow D[x] \rightarrow D[x] / \sim_{P=Q} \rightarrow D_{P=Q}, \quad \bar{\varphi}_{P=Q}: D \hookrightarrow D(x) \rightarrow \bar{D}_{P=Q},
$$

где $\sim_{P=Q}-$ конгруэнция на $D[x]$, порожденная соотношением $P(x) \sim Q(x), D_{P=Q}$ - полутело частных полукольца $D[x] / \sim_{P=Q}, D(x)$ - полуполе частных полукольца $D[x]$ и $\bar{D}_{P=Q}$ - фактор полуполя $D(x)$ по конгруэнции $\approx_{P=Q}$, порожденной соотношением $P \approx Q$. Однако не всякая такая конструкция дает расширение полуполя $D$; так, образ полуполя $D$ в $D_{x^{2}+1=a x}$ изоморфен $\mathbb{B}$ при любом $1>_{+} a \in D$.

Пусть $P(x)=a$ - мономиальное уравнение, $P(x)=a_{n} x^{n}+\cdots+a_{1} x$. Рассмотрим множество $D^{\mathbb{N}}$ всех последовательностей элементов $D$. Оно является $D[x]$-полумодулем, $x$ действует как сдвиг вправо. Положим $\left(d_{i}\right) \equiv\left(d_{i}^{\prime}\right)$, если $\exists N: \forall k>N d_{k}=d_{k}^{\prime}$; тогда $\equiv-$ конгруэнция. Обозначим $M_{D[x]}=D^{\mathbb{N}} / \equiv$. Образ последовательности $\left(d_{i}\right)$ относительно канонической сюръекции обозначим через $\left[d_{i}\right]$.

Рассмотрим в $M$ подмножество

$$
\bar{M}=\left\{\left[d_{i}\right] \in M: P(x)\left[d_{i}\right]=a\left[d_{i}\right]\right\} .
$$

Работа выполнена при частичной поддержке Российского фонда фундаментальных исследований (грант № 00-15-96128). 
Ясно, что $\bar{M}-D[x]$-подполумодуль, являющийся также $D[x] / \sim_{P=Q}$-полумодулем.

В $\bar{M}$ лежат $n$ (ненулевых) элементов $\left[e_{k}^{(i)}\right], i=1, \ldots, n$, задаваемых следующим образом: $e_{i}^{(i)}=1, e_{k}^{(i)}=0$ при $k=1, \ldots, n, k \neq i ; e_{k}^{(i)}=\sum_{j=1}^{n} a_{j} e_{k-j}^{(i)}$ при $k>n$. Более того, $M$

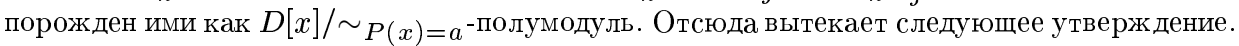

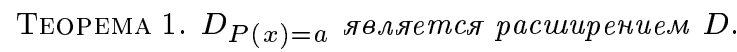

СледСтвиЕ 1. Для любого полуполя D существует его (универсальное) мономиальное алгебраическое замыкание $\widetilde{D}$.

Более подробное изучение структуры полумодуля $\bar{M}$ позволяет уточнить структуру расширения сократимого полуполя.

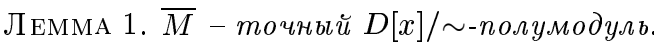

Теорема 2. Если $D$ сократимо, то и $D_{P(x)=a}$ сократимо.

Существуют сократимые полуполя, для которых расширение корнем мономиального уравнение неединственно. Примером может служить уравнение $x^{2}=2$ надполуполем $D=\mathbb{Q}_{+}[-\sqrt{2}] \cap$ $\mathbb{R}_{+}$: полуполя $\mathbb{Q}_{+}[-\sqrt{2}]$ и $\mathbb{Q}_{+}[\sqrt{2}]$ являются расширениями $D$ корнем этого уравнения и неизоморфны над $D$. Однако можно выделить класс сократимых полуполей, для которых это не так.

ТЕОРема 3. Мономиальное алгебраическое замыкание любого (сократимого) полуполя вида $D \cong F \cap \mathbb{R}_{+}$, әде $F-$ подполуполе в $\mathbb{R}_{+}$, единственно.

Г. Б. Шпиз [5] показал, что для мономиальной алгебраической замкнутости идемпотентного полуполя достаточно разрешимости любого уравнения вида $x^{n}=a$; при этом корень уравнения $a_{n} x^{n}+\cdots+a_{1} x=1$ задается формулой $x=\bigwedge_{k=1}^{n} a_{k}^{-1 / k}$. Опираясь на это, Е. М. Вечтомов и А. В. Ряттель [6] показали, что мономиальное алгебраическое замькание идемпотентного полуполя существует и единственно. Используя технику работы с дифференциалом, можно усилить эти результаты следующим образом.

ТеОРема 4. Пусть каждое уравнение вида $x^{n}=$ а разрешимо в D. Тогда любое строгое уравнение $P(x)=Q(x)$ разрешимо в $D$, при этом его решение представляется в виде $x=\bigwedge_{i} \bigvee_{j} x_{i j}=\bigvee_{j} \bigwedge_{i} x_{i j}$, где $x_{i j}$ - корень уравнения $p_{i} x_{i j}^{i-j}=q_{j}, p_{i} \neq 0, q_{j} \neq 0$.

СледствиЕ 2. Мономиальное алгебраическое замыкание идемпотентного полуполя является его строго алгебраическим замыканием. Расиирение любого идемпотентного полуполя корнем любого строгого уравнения $P(x)=Q(x)$ существует и единственHo.

Автор благодарен В. Т. Маркову за внимание к работе.

\section{СПИСОК ЛИТЕРАТУРЫ}

[1] И. И. Богданов. Об аддитивной структуре полутел // Вестн. Моск. ун-та. Сер. 1. 2004. №1 (в печати). [2] И. И. Богданов. Теорема коммутативности для полутел // Вестн. Моск. ун-та. Cеp. 1. 2004. № 3 (в печати). [3] J. S. Golan. The Theory of Semirings with Applications in Mathematics and Theoretical Computer Science. Harlow: Pitman, 1992. [4] H. J. Weinert // Acta Math. Acad. Sci. Hungar. 1962. V. 13. № 3-4. P. 365-378. [5] Г. Б. Шпиз // УМН. 2000. T. 55. № 5. С. 185-186. [6] Е. М. Вечтомов, А. В. Ряттель // Тезисы докладов V Международной конференции “Алгебра и теория чисел: современные проблемы и приложения". Тула, 2003. C. $68-69$.

Московский государственньй 